\title{
Roughness-Adaptive 3-D Watermarking Based on Masking Effect of Surface Roughness
}

\author{
Kwangtaek Kim, Member, IEEE, Mauro Barni, Senior Member, IEEE, and Hong Z. Tan, Senior Member, IEEE
}

\begin{abstract}
We present a method to improve watermark robustness by exploiting the masking effect of surface roughness on watermark visibility. Our idea is to adapt watermark strength to local surface roughness based on the knowledge that human eyes are less sensitive to changes on a rougher surface patch than those on a smoother surface. In order to quantify human sensitivity to surface roughness of polygonal meshes, we conducted a rigorous psychovisual experiment to obtain human watermark detection thresholds as a function of surface roughness. The results can be used to adaptively select watermark strength according to local surface roughness during the watermark embedding process. To test our idea, we applied it to the modified versions of two popular 3-D watermarking methods, one proposed by Benedens and one by Cayre and Macq. Experimental results showed that our approach improves watermark robustness as compared to the original algorithms. Further analyses indicated that the average watermark strength allowed by our roughness-adaptive method was larger than that by the original Benedens's and Cayre and Macq's methods while ensuring watermark imperceptibility. This was the main reason for the improved robustness observed in our experiments. We conclude that exploiting the masking property of human vision is a viable way to improve the robustness of 3-D watermarks, and can potentially be applied to other 3-D digital watermarking techniques.
\end{abstract}

Index Terms-Masking effect, polygonal mesh, robustness, roughness-adaptive 3-D watermarking, surface roughness.

\section{INTRODUCTION}

W ITH advances of computer graphics technology, 3-D digital contents have become increasingly popular in many applications such as video games, computer-aided design (CAD), virtual reality (VR), television broadcasting, and medical imaging. Through the internet access, 3-D digital contents are getting widely distributed or manipulated, often without copyright protection. For this reason, developing watermarking algorithms for 3-D polygonal meshes has received more interest than before. Compared to 2-D digital watermarking, however,

Manuscript received March 10, 2010; revised August 12, 2010; accepted August 12, 2010. Date of publication August 19, 2010; date of current version November 17, 2010. The work of K. Kim and H. Z. Tan was supported in part by the U.S. National Science Foundation under Grant 0836664 . The work of M. Barni was supported in part by the Italian Ministry of Research and Education under FIRB project RBIN04AC9W. The associate editor coordinating the review of this manuscript and approving it for publication was Dr. Mark (Hong-Yuan) Liao.

K. Kim and H. Z. Tan are with Haptic Interface Research Laboratory, Purdue University, West Lafayette, IN 47907-2035 USA (e-mail: samuelkim@purdue. edu; hongtan@purdue.edu).

M. Barni is with the Department of Information Engineering, University of Siena, 53100, Siena, Italy (e-mail: barni@dii.unisi.it).

Color versions of one or more of the figures in this paper are available online at http://ieeexplore.ieee.org.

Digital Object Identifier 10.1109/TIFS.2010.2068546
3-D watermarking is more difficult due to the increased complexity associated with arbitrary shapes. Three-dimensional watermarks are more fragile due to the various ways in which they can be destroyed by simply altering the meshes making up the 3-D objects. Therefore, existing 2-D watermarking techniques cannot be directly applied to 3-D models, thereby necessitating new approaches that are specifically designed for 3-D objects.

The challenge is to design 3-D digital watermarks that are unobtrusive (transparent), robust, and space efficient (capacity) [3]. The unobtrusive requirement means that the embedded watermark should not interfere with the intended use of a model, which may imply imperceptibility. Robustness refers to the ability for the watermark to survive various intentional and nonintentional attacks to the watermarked 3-D model. This is a very challenging requirement as no algorithm has been shown to be perfectly robust. However, constant improvements are being made that result in more robust watermarking schemes as compared to previous methods. The last requirement is about having enough space for watermark embedding. To meet all three requirements at the same time is not trivial.

Of the three requirements, unobtrusiveness and robustness conflict with each other. From an unobtrusiveness perspective, watermark strength should be as small as possible. From a robustness perspective, however, watermark strength should be large so that the watermark can not be easily destroyed. It is a trade-off to satisfy both requirements at the same time.

In an effort to improve watermark robustness while maintaining its imperceptibility, researchers have developed perceptual coding techniques, that exploit human visual perception, and in particular the masking effect typical of the human visual system. Masking refers to our decreased ability to perceive a stimulus (e.g., a watermark) in the presence of other signals (e.g., polygonal mesh). In the areas of image and video watermarking, various attempts have been made by utilizing luminance and frequency sensitivity, and contrast masking to improve the imperceptibility and robustness of watermarks [4]-[9]. Two-dimensional watermarking techniques taking into account human sensitivity to luminance, frequency, and contrast are more effective at improving both robustness and unobtrusiveness as compared to classic 2-D watermarking schemes. It suggests that the same approach can be applied to 3-D watermarking with similar expected improvements. Doing so requires that we have a way to specify human sensitivity to surface variations as a function of local geometric properties, such as 3-D surface roughness.

In the present study, we introduce a new approach for adaptively adjusting 3-D watermark strength based on local surface roughness. Our work takes advantage of a recent study by Corsini et al. [10] who introduced a method to estimate surface 
roughness of polygonal meshes for assessing visual distortions introduced by watermarking. The present study makes several contributions. First, we quantify human sensitivity to surface variations as a function of estimated local roughness by conducting psychovisual experiments. The result is a precise functional relationship between local roughness and the just noticeable difference (JND). This function is subsequently used in our roughness-adaptive watermarking scheme to ensure imperceptibility. Our approach is, therefore, more perception based as compared to previous attempts [11] that used the local characteristics of 3-D models to adapt watermark strengths without employing human sensitivity functions.

Second, using the experimentally derived JND versus local roughness relationship, we propose a roughness-adaptive method for ensuring locally maximal watermark strength (to improve watermark robustness) while maintaining the watermark's imperceptibility. Third, we evaluate our roughness-adaptive 3-D watermarking approach by applying it to two existing 3-D watermarking techniques, one proposed by Benedens [1], [12] and the other by Cayre and Macq [2], to demonstrate the effectiveness of our approach and to show that it can be applied to different watermarking schemes as long as the relationship between watermark strength and invisibility is estimated for the watermarking scheme at hand, by using the experimental methodology introduced in this paper.

Although there are many other watermarking schemes that could have been used for the evaluation of our approach, we chose these two particular methods for three reasons. First, both methods embed watermarks in the spatial domain, hence our research complements and extends an analysis carried out in [11] where roughness adaptive watermarking was addressed in the spectral domain. Second, the two methods have opposite characteristics in terms of robustness and capacity, thus they constitute good examples of the generality and extendability of our approach. More specifically, Benedens's method is a robust method with limited capacity, whereas Carye and Macq's method provides good capacity but is less robust due to the dependency on mesh connectivity. Third, the artifacts introduced by the two watermarking schemes are very different. Using both methods, therefore, demonstrates the generality of our approach.

We also introduced several modifications and improvements to Benedens's and Cayre and Macq's methods in order to improve the methods themselves and to make them receptive to our roughness-adaptive 3-D watermarking approach.

The remainder of this paper is organized as follows. In Section II, we present previous work, including a review of the two existing methods used for the evaluation of our roughness-adaptive watermarking scheme. In Section III, we describe the psychovisual experiment for estimating JND as a function of local surface roughness. Section IV describes our modifications to Cayre and Macq's method. Section V presents our roughness-adaptive watermarking approach. Evaluation results appear in Section VI. Finally, we conclude the paper in Section VII.

\section{PREVIOUS WORK}

In this section, we review previous approaches to satisfying the invisibility requirement in 3-D watermarking applications.

In image and video watermarking, it is well known that visual masking effect can be utilized in order to minimize visual dis- tortion introduced by watermarks while maximizing watermark strengths and hence improve robustness [4]-[7], [9]. As an extension to polygonal meshes, Ferwerda et al. [13] analyzed how the presence of one visual pattern affects the detectability of another. They demonstrated that the pattern of 3-D textures can be flexibly selected and used to mask faceting artifacts caused by polygonal tessellation of a curved surface. This fact has encouraged researchers working in 3-D watermarking to exploit the masking effect in several ways.

Kanai et al. [14] exploited the fact that the human vision system (HVS) is less sensitive to changes in high-frequency areas of polygonal meshes. They analyzed an input mesh with a wavelet transform and modulated the high-frequency components to embed the watermarks. Similarly, Bors et al. [15] used human detection thresholds on local mesh variations to choose the surface regions where the human eye is less sensitive to changes. Only those chosen vertices were modified by the watermarks.

The key differences between our method and the above two methods are that both Bors et al. and Kanai et al. used a constant watermark strength in surface areas where humans are less sensitive to watermark embedding, and neither method embedded watermarks in surface areas where humans are more sensitive to changes. These methods, therefore, adapted watermark strengths in a coarse way (either ON or OFF) without fully exploiting the way the HVS perceives the watermark. On the contrary, our method allows a continuous optimization of watermark strengths on a vertex by vertex basis.

In a study that somewhat resembles our approach, Uccheddu et al. [11] applied masking effect of surface roughness to a wavelet-based 3-D watermarking method, which was previously developed by the same authors [16], to diminish the degradation of the host mesh. In the beginning stage of watermark embedding process, the host signal is decomposed in a multiresolution framework with wavelet coefficients representing surface details by means of the algorithm proposed by Lounsbery et al. [17]. Subjective experiments were conducted to estimate watermark detection threshold as a function of local roughness values. Watermarks were embedded by modifying the wavelet coefficients at a given level of resolution. Triangles to be modified were selected by using the experimentally derived roughness-based threshold; only those vertices for which the local roughness was greater than the threshold were watermarked.

Despite the apparent similarity between our present study and [11], there are several important differences, concerning how watermark strength is selected and which surface areas are chosen for watermarking. In our approach, the watermark strength is selected automatically based on a human perception curve determined by psychovisual experiments, while in [11] the strength was adjusted manually. In addition, our method [18] allows watermark strength to be adjusted on a continuous scale on a vertex by vertex basis, while in [11] each vertex was either modified with a fixed-strength watermark or it was not altered at all.

The present study extends the approach described in our previous research [18]. In the previous study, the basic idea was to adjust watermark strength according to the local surface roughness. The idea was then applied to Benedens's watermarking 
method [1], [12], showing improvements in terms of roughness and invisibility. The novelties introduced in the present study include: 1) refinement of the psychovisual experiments to take into account several types of watermarking disturbances; 2) a more rigorous definition of the functional relationship between admissible watermark strength and surface roughness; 3) application of roughness-adaptive watermarking to Cayre and Macq's method whose characteristics are complementary to those of Benedens's algorithm; and 4) evaluation of the results on a larger number of 3-D models.

\section{A. Overview of Two Watermarking Schemes}

Throughout our research, we considered two different 3-D watermarking schemes with complementary characteristics: one developed by Benedens [1], [12] and the other proposed by Cayre and Macq [2]. As it will become evident later on in this paper, these two schemes introduce quite different disturbances to the original meshes, hence permitting us to evaluate the performance of roughness-based watermarking scheme under different conditions and to demonstrate its generality. In the remainder of this section, we give a brief overview of these two watermarking schemes, and describe a few modifications we introduced in order to make them more receptive to roughness-based adaptation of watermark strength.

1) Benedens's Method: Benedens's nonblind, geometry-based 3-D watermarking method [1] uses the distribution of face normals on polygonal meshes for watermark embedding. In Benedens's method, the watermark is embedded by modifying any of the following three features: i) the mean of normals, ii) the mean angle between normals and bin center (BC) normal, or iii) the amount of normals in a bin. In this work, we considered the third feature since it provides the most straight-forward way to adjust watermark strength. Considering only the third feature, the embedding process of Benedens's method is summarized below:

1) Create a unit sphere, and then tessellate the surface of the unit sphere to generate bins defined by a BC normal and a bin angle $\left(\varphi^{R}\right)$ (also referred to as bin radius). The same bin radius is used for all the bins. Bins are cone-shaped as illustrated in the left image of Fig. 1.

2) Randomly choose a set of bins for embedding watermark bits and for sampling face normals. A face normal is assigned to a bin if the angle formed between the face normal (BP) and the BC normal (Fig. 1, left image) is smaller than that formed between the cone that passes through its apex.

3) For each bin, compute the ratio of normals $\left(n k_{i}\right)$ inside the bin kernel predefined by a kernel angle $\varphi^{k}$ over all normals inside the bin. The 2-D projected kernel area is shown as the gray inner circle in the right image of Fig. 1.

4) Transform the 3-D face normals in each bin into 2-D coordinates in the $X_{1}$ and $X_{2}$ plane (see the left image of Fig. 1) and perform the core embedding process as described below.

During the core embedding process, watermark bits are inserted by changing the number of normals inside the kernel area in each bin. Specifically, to embed a bit "0", all the normals outside the kernel are moved inside the kernel as depicted in the right image of Fig. 1. It means that $n k_{i}$ (the ratio of normals inside the

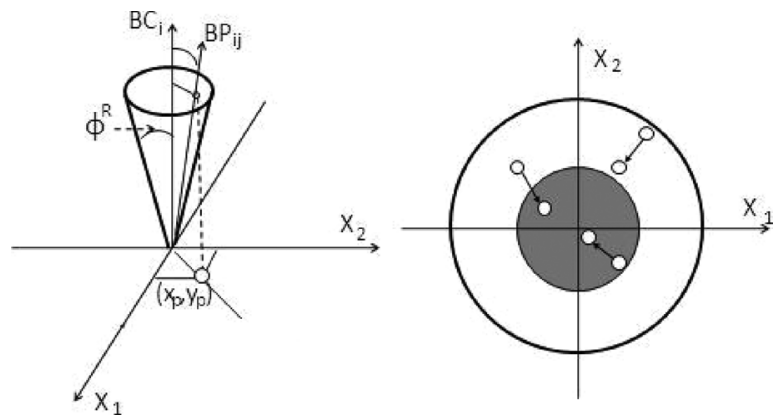

Fig. 1. (left) Transformation of 3-D coordinates into 2-D coordinates. $\varphi^{R}$ is a bin angle, and $\mathrm{BC}_{i}$ and $\mathrm{BP}_{i j}$ are a $\mathrm{BC}$ normal and a sampled normal, respectively, where $i$ and $j$ denote the index of bins and sampled normals. (right) Embedding a bit " 0 " by pushing normals into the kernel area. Modified from [1].

kernel) becomes 1.0, which is the maximum for any bin. Conversely, a bit " 1 " is embedded by taking all the normals inside the kernel out of the kernel so that $n k_{i}$ goes to 0.0 , the minimum for any bin. Finding the best normal direction for embedding is performed by an optimization algorithm called the Downhill simplex method which is also called the Nelder-Mead method [19]. The Downhill simplex method is a nonlinear optimization technique using a simplex to approximate a local optimum of a problem with $n$ variables. According to the optimizing process, an initial watermark strength value, flexibly chosen according to the object size (see [1]), is optimized.

The two cost functions, defined in (1) and (2) for bits 0 and 1 , respectively, are used as an objective function for the optimization. In the equations, $S_{i}$ represents a watermark code to be embedded, where $i$ is a bin index. The notation $f, v \rightarrow v^{\prime}$ means that an objective function returns a new vertex $v^{\prime}$ from an old vertex $v$ by minimizing the cost function. As an example of how the cost functions work, let us consider the case in which a bit $\left(S_{i}=0\right)$ is embedded. The cost function in (1) returns a new vertex position $v^{\prime}$ when the angle difference between the normal vector $\left(\mathrm{BP}_{i j}^{\prime}\right)$ of the new vertex and $\mathrm{BC}$ normal $\left(\mathrm{BC}_{i}\right)$ is minimized. Thus a successful candidate for a new vertex is returned only when the $\mathrm{BP}_{i j}^{\prime}$ becomes as close as possible to the $\mathrm{BC}_{i}$. More details about the two cost functions can be found in [1], [12]

$$
\begin{aligned}
\operatorname{cost}_{f, v \rightarrow v^{\prime}} & =\frac{\cos ^{-1}\left(\left\langle\mathrm{BP}_{i j}^{\prime}, \mathrm{BC}_{i}\right\rangle\right)}{\varphi_{i}^{R}}, \quad S_{i}=0 \\
\operatorname{cost}_{f, v \rightarrow v^{\prime}} & =\cos \left[\frac{\pi}{2}\left(\frac{\cos ^{-1}\left(\left\langle\mathrm{BP}_{i j}^{\prime}, \mathrm{BC}_{i}\right\rangle\right)}{\varphi_{i}^{R}}\right)\right], \quad S_{i}=1 .
\end{aligned}
$$

In order to minimize the distortion of the surface of the input model, the following constraints are imposed during the embedding process:

1) The normal of a face adjacent to a vertex $v$ in the bin is not allowed to change by an angle that is larger than or equal to $\alpha$.

2) The normal of a face adjacent to a vertex $v$ that is not in the bin is not allowed to change by an angle that is larger than or equal to $\beta$.

3) No normal is allowed to leave its bin. 
For watermark retrieval, the information about bins (bin radius, kernel radius, the ratio of normals in each bin, and the chosen bins used in the embedding process) need to be delivered to the extraction stage. With the watermarked polygonal mesh, repeat the same steps (1-4) of the embedding process. Then the ratio of normals $n k_{i}$ in each bin is compared with the original value of $n k_{i}$. If $n k_{i}$ of the watermarked mesh is larger than the original value $n k_{i}$, then the embedded watermark bit is a "0". Otherwise, it is a " 1 ".

Watermarks embedded by Benedens's method are especially robust against mesh-simplification and vertex randomization, because the distribution of face normals is approximately invariant to these kinds of modifications of the polygonal meshes.

Two drawbacks of Benedens's watermarking algorithms exist: 1) the need to carry the original $n k_{i}$ values to the retrieval stage, and 2) the intrinsic weakness of the "1" bits. In the present study, we used a modified version of Benedens's scheme introduced in our earlier study [18], which is briefly summarized below.

a) A Blind Version of Benedens's Method: Retrieval of a watermark embedded by Benedens's method requires the availability of a priori knowledge including bin radius, number of bins, and the original ratio of normals $\left(n k_{i}\right)$ in the kernel of each bin. This information constitutes the secret key needed to retrieve the watermark. Since the original values of $n k_{i}$ depend on the polygonal mesh of the 3-D object, Benedens's method may not be considered a truly blind watermarking technique. To eliminate the need to carry the original $n k$ values to the watermark extracting stage, we proposed to use the probability distribution of normals in the kernel area of each bin [18]. The main idea was to choose the kernel radius in such a way that, on average, the ratio of face normals inside the kernel is a fixed value. More details can be found in [18].

b) Improvement to Bit "I" Robustness: In the original Benedens's method, during the embedding process, the normals are moved in two opposite directions. When embedding a bit "0", all normals in the bin are moved inside the kernel area [the dark inner circle in Fig. 2(a)]. When embedding a bit "1", however, the normals are moved towards the border of the bin and are pushed as close to the rim of the bin as possible. There are, therefore, two imaginary embedding zones: one around the $\mathrm{BC}$ and the other around the rim of the bin. The problem, however, is that the normals located on the rim of the bin can be easily pushed out of the bin. Therefore, bit 1 is less robust than bit 0 . To improve the robustness of bit " 1 ", the ideal embedding zone has to be moved away from the bin rim. The new embedding zone defined by the dashed circle in the right image of Fig. 2(a) was defined by a new radius $\varphi^{k 1}$ such that the surface area of the spherical cap was $3 / 4$ of that of the bin. In searching for a safer area for embedding " 1 " bits, it was desired that the dashed circle was neither too close to the dark inner circle (otherwise normals embedded with "1" bit can be easily pushed into the "0" bit zone during an attack) nor to the outside border of the bin (so that normals cannot be easily pushed outside of the bin during an attack). A good compromise, therefore, was for the dashed circle to be midway between the border of the dark inner circle and the border of the bin. With this placement of the dashed circle, the corresponding surface area of the spherical cap was $3 / 4$ of that of the bin. In our previous study [18], the error rate of bit "1" was reduced from $15 \%$ to $10 \%$ as

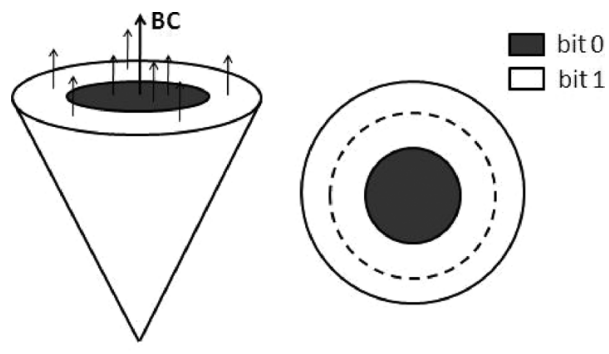

(a)

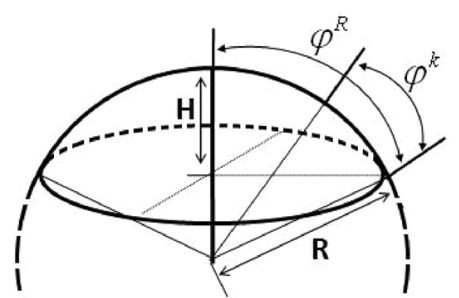

(b)

Fig. 2. (a) Two views of a bin with sampled normals. The dark area (inner circle) is the kernel area defined by $\varphi^{k}$, which is used for embedding " 0 " bits. The bin area excluding the dark area is used for embedding " 1 " bits. The dashed circle represents the new zone for embedding "1" bits. (b) A sphere cap (a bin) defined by $\varphi^{R}$. $R$ and $H$ represent radius of the sphere and height from the top of the cap to the bottom of the base circle, respectively.

compared to an error rate of $11 \%$ for bit " 0 ", indicating that the new kernel achieved a more balanced robustness between bit "1" and bit " 0 ". More details, including improved robustness results, can be found in [18].

2) Cayre and Macq's Method: Cayre and Macq's method builds upon the basic idea of triangle strip peeling sequence (TSPS) that encodes a payload by moving over a triangular surface mesh. With this method, each triangle always has one entry edge and two possible exit edges, as seen in Fig. 3(a). The watermarking algorithm requires two main steps as described below.

1) Generate a list of consecutive triangles of the mesh: The list of triangles is established as seen in Fig. 3(b). The list of triangles is stored as a secret key to be carried to the extractor. The length of the key must be the same as that of the list of admissible triangles required to convey the payload.

2) Construct a macro embedding procedure (MEP): Each triangle has two states defined by the position $P(C)$ of the orthogonal projection of the triangle summit $C$ on the entry edge $A B$. The entry edge $A B$ is divided into two subsets $S_{1}$ ("1") and $S_{0}$ ("0"), as seen in Fig. 4(b). If $P(C) \in S_{0}$, then the triangle is in a "0" state; otherwise, $P(C) \in S_{1}$, and the triangle is in a " 1 " state. For every triangle, there are two possible cases: 1) $P(C) \in S_{i}$, and no modification is needed; or 2) $P(C) \notin S_{i}$, then $C$ is move to $C^{\prime}$ so that $P\left(C^{\prime}\right) \in S_{i}$ [see Fig. 4(b)], where $i$ is 0 or 1 .

The value of $\lambda$ [seen in Fig. 4(b)] has to be small enough to avoid visual degradation of the mesh, but large enough to allow accurate payload detection. The parameter $n$ [see Fig. 4(a)] controls the smoothness of the algorithm. As $n$ increases, $\lambda$ decreases and the amount of distortion to be introduced gets smaller. On the other hand, as $n$ increases, bit retrieval errors also increase due to the decrease in the interval size. This leads to reduced robustness. 


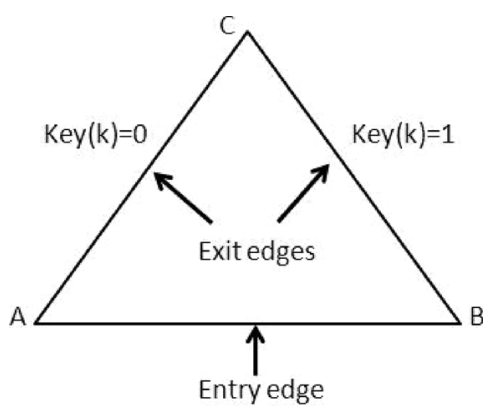

(a)

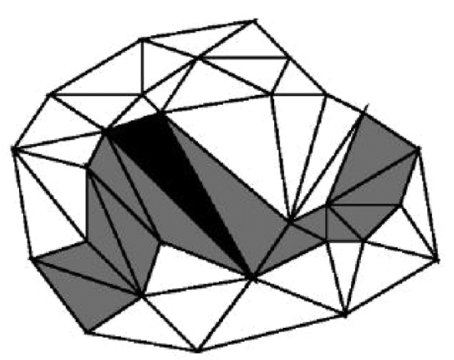

(b)

Fig. 3. (a) Triangle to be traversed for watermarking by Cayre and Macq's method. (b) Example of a list of triangles generated by a secrete key. The triangle strip peeling sequence (TSPS) path is in gray and the cell to be processed is in black. From [2, Fig. 2].

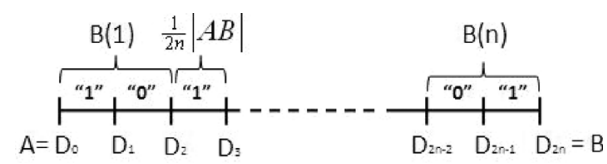

(a)

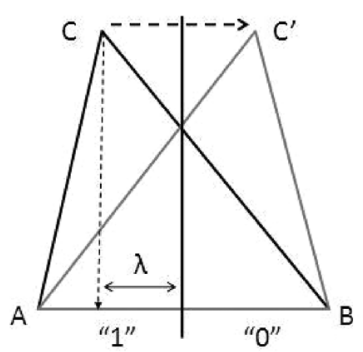

(b)

Fig. 4. (a) Decomposition of the entry edge $A B$ into two interleaved subsets with the $2 n$ binary values. (b) Example of the first-order Macro Embedding Procedure [(MEP) $n=1$ ] encoding. Modified from [2, Fig. 4].

\section{WATERMARK PERCEPTIBILITY AS A FUnCTION OF SURFACE ROUGHNESS}

In this section, we present the psychovisual experiment that we designed and carried out to derive the functional relationship between local surface roughness and the maximum watermark strength that can be used while maintaining watermark invisibility. Specifically, human detection thresholds for the perceptibility of geometrical surface distortions were estimated for three watermarking techniques: a modified version of Benedens's method (see Section II), a modified version of Cayre and Macq's method (see Section IV), and watermarks consisting of the additive Gaussian noise to vertex positions. It was conceivable that the threshold curves for the three methods would be quite different since the strategies to embed watermarks are all different. If this turned out to be the case, then a different rule should be used to adjust watermark strength according to

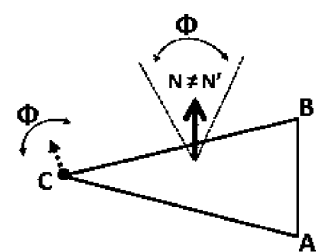

(a)

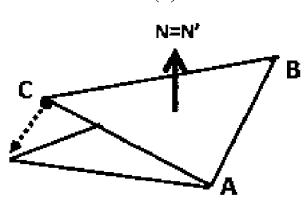

(b)

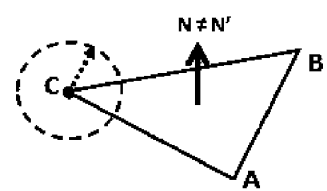

(c)

Fig. 5. Three different strategies for watermark embedding on a triangle. $N$ and $N^{\prime}$ are the normals before and after embedding. (a) Geometrical change of a triangle by Benedens's method with a bin radius $\phi$. (b) In-plane vertex distorted by Cayre and Macq's method. (c) Watermarking with additive Gaussian noise. The dashed circle around the vertex $C$ represents the projection of a sphere for the range of allowable changes.

the watermarking method. On the other hand, if the threshold curves were similar for the three watermarking techniques, then the same adaptation rule could be used regardless of the watermarking technique.

The three different methods were selected because they differ in the directions along which vertices are modified with respect to the normals of surface mesh. Benedens's method perturbs vertex normals by changing the locations of the vertices that belong to a bin with a radius $\phi$ [see Fig. 5(a)]. The direction of the vertex change is the same as the direction of normal change. The range of allowable changes is limited by the size of the bin radius $\phi$. The distortion made by Cayre and Macq's method, on the contrary, is in a direction perpendicular to the normal of a triangular mesh [see Fig. 5(b)]. The new vertex $C$ lies on a line parallel to the triangle edge $A B$. In this case, the normal $N$ is always preserved during the perturbation. Using additive Gaussian noise, a vertex is changed in a randomized direction [see Fig. 5(c)].

In summary, both Benedens's method and additive Gaussian noise alter the normals of triangular meshes whereas Cayre and Macq's method does not. Additive Gaussian noise can also result in larger distortions than Benedens's method since changes to the vertex $(C$ in Fig. 5) is unlimited in its direction. We were interested in comparing human threshold curves for Benedens's method and additive Gaussian noise, as well as comparing the curves for these two methods to that of Cayre and Macq's method.

\section{A. Methods}

The psychovisual experiment was designed to estimate the relation between visual watermark detection threshold (in terms of watermark strength generically indicated by $\delta$ ) and local roughness of spherical surfaces. The watermark strength $\delta$ is controlled differently in the three watermarking methods since the geometrical properties used for watermarking are different. For 


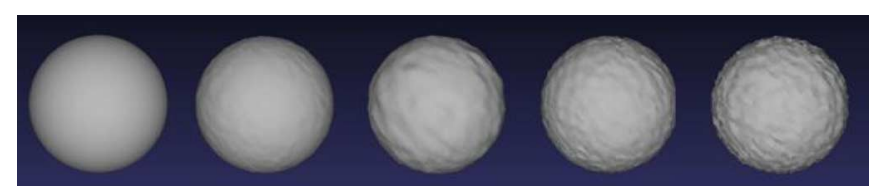

Fig. 6. Five reference spherical surfaces used in the present psychovisual experiments. The estimated roughness values were, from left to right, 0.000082 , $0.001704,0.003139,0.010937$, and 0.025304 , respectively. The five surfaces contained the same number of vertices (3752) and faces (7500).

instance, in Benedens's method control of watermark strength is obtained by restricting the search space in the Downhill Simplex optimization method. In Cayre and Macq's method, watermark strength is not easily modifiable since it is affected by the partition size determined by the order of MEP. We, therefore, modified the original Cayre and Macq's method to make the watermark strength easily modifiable for our experiments (see details in Section IV). The watermark strength for additive Gaussian noise method corresponds to the amount by which a vertex is altered in a randomized direction. In the present study, $\delta$ is expressed as a normalized value over the diagonal length of the bounding box of a 3-D object.

\section{B. Participants}

Ten participants (five males and five females) took part in the experiment. None of the participants reported any visual deficiencies.

\section{Stimuli}

The visual display consisted of a spherical surface rendered with 3752 vertices and 7500 faces. The number of vertices and faces were chosen so that real-time rendering could be achieved during the experiment. The image of the sphere occupied a visual angle of roughly $30^{\circ}$. Five reference spherical surfaces with different roughness levels were created. Roughness was controlled by perturbing the vertices with additive Gaussian noise. Specifically, the roughness level was specified by the variance of a Gaussian probability distribution function $N(0, \sigma)$ that generated the additive noise. The direction of the additive noise was chosen randomly. Fig. 6 shows the five reference stimuli with increasing surface roughness. The left-most sphere has a smooth surface with no additive noise. The roughness level of each spherical surface was estimated with a one-ring roughness measure based on the multiscale roughness estimation method proposed by Corsini et al. [10]. The estimated roughness for the five reference surfaces were 0.000082, $0.001704,0.003139,0.010937$, and 0.025304 , respectively. These values were chosen by a pilot study where more points were chosen for the part of the perception curve that changed rapidly (i.e., near the lowest roughness values).

Each of the three watermarking schemes was applied to each of the five reference surfaces to obtain human detection thresholds for visual watermarks. Due to the time required to generate watermarked surface using Benedens's method, the stimuli for Benedens's method were precomputed. The stimuli for the other two watermarking methods were computed in real time.

The parameters used for the Benedens's method were $\varphi^{R}=$ $10^{\circ}, 10$ bins, and no $\beta$. For Cayre and Macq's method, a list of triangles was randomly generated for embedding the watermark

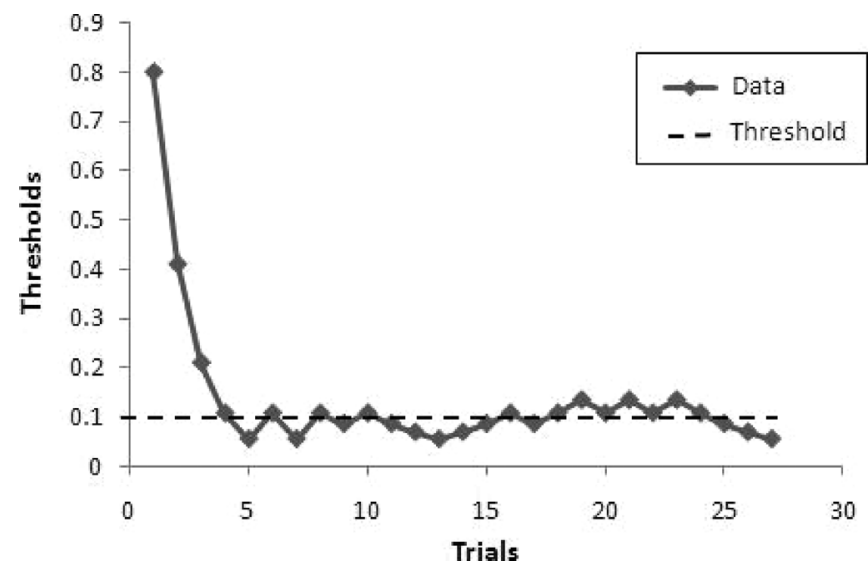

Fig. 7. One-up one-down (1U1D) representative data plot the one-up one-down adaptive procedure. The data converge around the threshold indicated by the dashed line.

and the vertices of the list of triangles were altered by the modified version of Cayre and Macq's method enabling roughness adaptation (see also Section IV).

For the watermarking method using additive noise, Gaussian noise was used to randomly alter chosen vertices of the spherical surface in a random direction, as specified below

$$
\vec{v}_{\text {new }}(i)=\vec{v}(i)+\delta * \overrightarrow{n_{r}}(i)
$$

where $\vec{v}_{\text {new }}(i)$ is the modified vector of the $i$ th vertex $\vec{v}(i)$, $\delta$ denotes the watermark strength that varied according to the correctness of participant's responses, and $\overrightarrow{n_{r}}(i)$ is a random unitary normal vector.

\section{Procedures}

A three-interval forced-choice (3IFC) one-up one-down adaptive procedure [20] was used to measure watermark detection thresholds as a function of surface roughness. The threshold so obtained corresponded to the 50 percentile point on the psychometric function. On each trial, the participant looked at three spherical surfaces, two reference surfaces (without watermarks) and a test surface (with watermarks), presented on a 17-in TFT PC monitor in a dark room. The spherical surfaces were rendered by Gouraud shading [21] with directional lighting developed with OpenGL. The position of the watermarked surface was randomly chosen to be on the left, middle, or right of the monitor on each trial. The participant's task was to indicate which spherical surface looked different (i.e., contained the watermark). According to the one-up one-down adaptive rule (see Fig. 7), the stimulus intensity $(\delta)$ was increased after an incorrect response and decreased after a correct response. The initial $\delta$ value was chosen to be large enough so that the test surface looked clearly different from the reference surface. The value of $\delta$ then decreased or increased by a fixed step size $(6 \mathrm{~dB})$, depending on the participant's responses. After three initial reversals (a reversal occurred when the value of $\delta$ decreased after increasing, or vice versa), the value of $\delta$ changed by a smaller step size $(2 \mathrm{~dB})$. The initial larger change in $\delta$ was necessary for faster convergence of the $\delta$ values, whereas the later smaller change in $\delta$ improved the resolution of threshold estimates. The adaptive series was terminated after 12 reversals at the smaller step size. The detection 


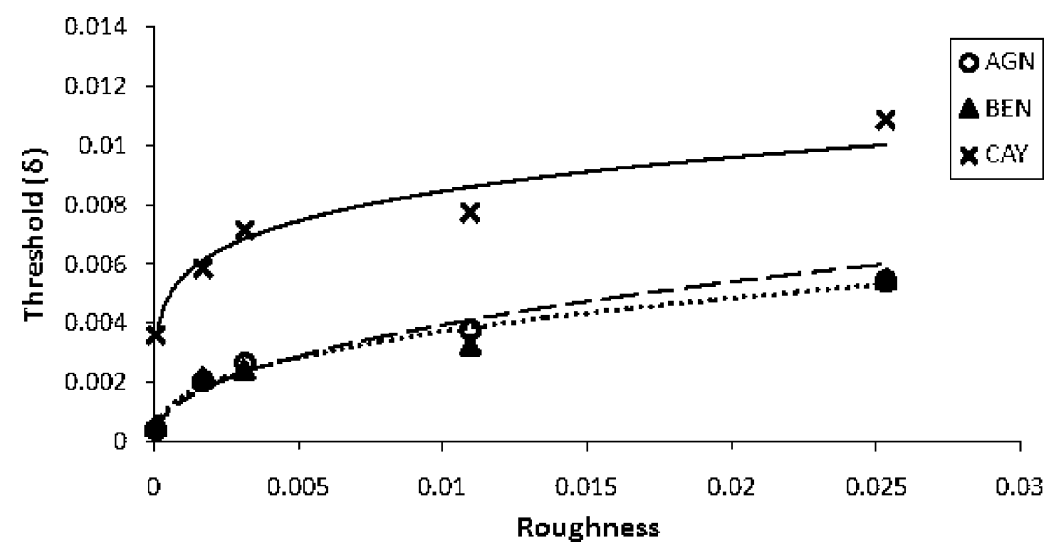

Fig. 8. Power regression models of human sensitivity to watermarks for the three watermarking schemes. See texts for details.

threshold was computed by taking the average of the $\delta$ values from the last 12 reversals. Each participant was tested once per combination of reference surface roughness and watermarking method, resulting in a total of 15 adaptive series ( 5 references $\times 3$ watermarking techniques) per participant. It took about one and a half hours for each participant to finish all the 15 series.

\section{E. Results}

The average detection thresholds for the ten participants are shown in Fig. 8. For each watermarking method, thresholds followed a monotonically increasing trend as surface roughness increased. The thresholds with Cayre and Macq's method are much larger than those with Benedens's method and additive Gaussian noise, indicating that stronger watermarks can be embedded with Cayre and Macq's method. The results suggest that humans are more sensitive to changes in the direction of normals than to changes of vertices in a plane that is perpendicular to face normals. It is also apparent that the thresholds for Benedens's method and additive Gaussian noise were very similar, suggesting that the range of directions along which vertex $C$ can be altered (see Fig. 5) does not have a strong effect on the perceptibility of watermarks.

The data shown in Fig. 8 were fit by power regression models with $R^{2}$ values ${ }^{1}$ of $0.9665,0.9841$, and 0.982 for Cayre and Macq's method (CAY), additive Gaussian noise (AGN), and Benedens's method (BEN), respectively. The three best-fitting power functions are as follows:

$$
\begin{aligned}
& \delta_{\mathrm{CAY}}=0.0195 * S^{0.1814} \\
& \delta_{\mathrm{AGN}}=0.0313 * S^{0.4499} \\
& \delta_{\mathrm{BEN}}=0.3851 * S^{0.3851}
\end{aligned}
$$

where $\delta_{\mathrm{CAY}}, \delta_{\mathrm{AGN}}$, and $\delta_{\mathrm{BEN}}$ denote watermark strengths for the respective watermarking techniques, and $S$ denotes local surface roughness.

The results show that in general the application of a roughness adaptive watermarking strategy requires that the dependence between visibility and watermark strength be evaluated for the particular watermarking scheme. On the other hand, the

\footnotetext{
${ }^{1}$ The $R^{2}$ value indicates how well a regression model approximates data points, where $R^{2}=1.0$ means a perfect fit. It is calculated by $R^{2}=\operatorname{Cov}(X, Y) /\{\operatorname{StdDev}(X) * \operatorname{StdDev}(Y)\}$.
}

similarity between the curves obtained from Benedens's (BEN) and additive Gaussian noise (AGN) methods seems to indicate that watermarking schemes could be grouped according to the type of modifications applied to the vertices, and that the same relationship between watermark strength and visibility could be used for schemes belonging to the same class. For instance, the same regression model can be used for Benedens's method and the additive Gaussian noise methods, since the best-fitting curves are quite similar in Fig. 8. This is an interesting research direction that we leave for future work. In the present study, we used (5) for both the Benedens's method and the additive Gaussian method, and used (3) for Cayre's and Macq's method. In Section V, we show how the regression models shown in (3) and (5) can be used to devise a roughness adaptive embedding rule.

\section{ModificATIONS TO CAYRE AND MACQ’s Method}

In the original watermarking algorithm proposed by Cayre and Macq, the watermark strength cannot be easily controlled. The watermark strength depends on the number of intervals into which the entry edge of the triangle is split, and the derivation from the number of intervals to watermark strength is not straightforward. For this reason, we modified Cayre and Macq's method to make it suitable for the incorporation of our roughness-based watermark-strength adaptation scheme. The rest of this section describes the modifications in detail.

\section{A. New Partition of the Entry Edge $A B$}

The limited freedom in handling the size of watermark strength with the original entry-edge decomposition described in Fig. 9(a) motivated us to propose a new decomposition of the entry edge $A B$ that makes it possible to control watermark strength [see Fig. 9(b)]. The key difference from the original Cayre and Macq's method was that two infinite sized intervals ( $I_{0}$ and $I_{3}$ ) were added to increase watermark strength, thereby guaranteeing improved watermark robustness. The entry edge $A B$ is extended in both directions into infinitely and divided into four intervals by $D_{0}, D_{1}$, and $D_{2}$ [see (6)], as seen in Fig. 9(b)

$$
\begin{aligned}
I_{k+1} & =\overline{D_{k} D_{k+1}}, \quad \text { for } k=0,1 \\
\left|I_{0 \text { or } 3}\right| & =\infty \&\left|I_{1 \text { or } 2}\right|=\frac{1}{2}|A B| .
\end{aligned}
$$




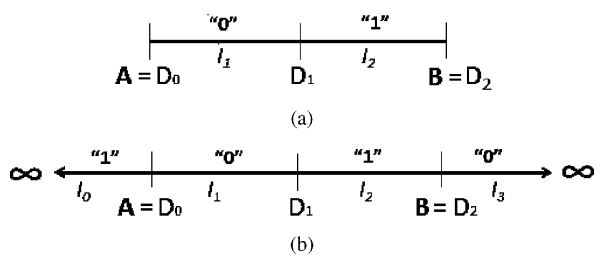

(b)

Fig. 9. Comparison of entry edge decomposition using the original Cayre and Macq's method and our modified version. (a) Original structure $(n=1)$, edited from [2]. (b) Our modified structure.
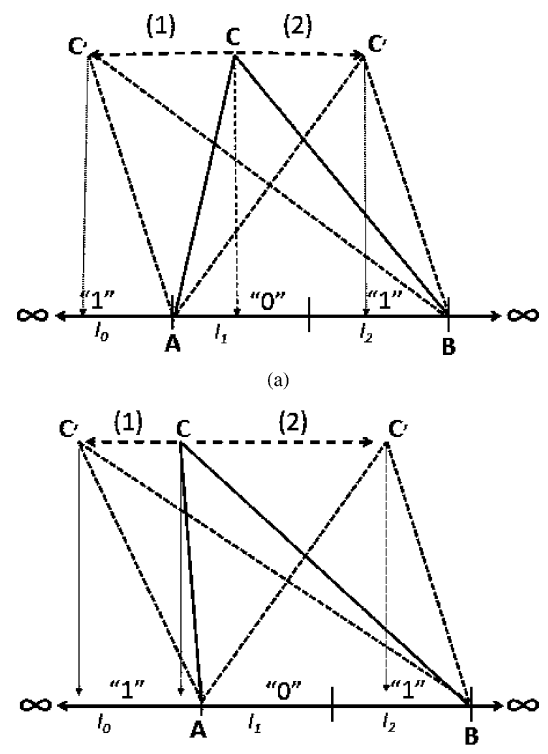

(b)

Fig. 10. Two examples of embedding. For each example, two candidate directions exist for the modification. (a) When $P(C) \notin S_{i}$. (b) When $P(C) \in S_{i}$.

\section{B. Embedding}

With the modified decomposition method, the position of the vertex $C^{\prime}$ (see Fig. 4) is changed for both cases of $P(C) \notin S_{i}$ and $P(C) \in S_{i}$. Recall that the original Cayre and Macq's method allows a symmetrical reflection of vertex $C$ to $C^{\prime}$ only when $P(C) \notin S_{i}$. Equation (7) shows how the new position of the vertex $C^{\prime}$ is calculated in our modified method

$$
C^{\prime}=C+\delta_{\mathrm{est}} \cdot \vec{N}
$$

where $C$ and $C^{\prime}$ were the current and new vertices, $\delta_{\text {est }}$ determines the watermark strength (see (10) later in Section V), and $\vec{N}=C^{\prime}-C$ is a unit vector parallel to the $A B$ edge.

Fig. 10 further illustrates how vertex $C$ is modified in both cases $P(C) \notin S_{i}$ and $P(C) \in S_{i}$. For simplicity, we discuss the embedding of bit " 1 ", since the extension to bit " 0 " is trivial. The watermark can be embedded in two different ways, hereafter called Method 1 and Method 2, respectively. By referring to Fig. 10, Method 1 enforces $C$ to move toward a position $C^{\prime}$ which belongs to $I_{0}$ of infinite size, whereas Method 2 moves $C$ toward a position $C^{\prime}$ in $I_{2}$ of limited size.

Although a modification can occur by either Method 1 or 2 depending on the value of $\delta_{\text {est }}$ (see Fig. 10), Method 1 is more desirable. This is because $I_{1}$ and $I_{2}$ are of equal sizes and divide the length of $A B$ evenly, whereas $I_{0}$ and $I_{3}$ are of infinite size and extend from $A$ and $B$ towards infinity. If $C$ can be moved further in $I_{0}$ towards infinity, then a superior robustness is obtained since it becomes much harder for an attack to push $C^{\prime}$

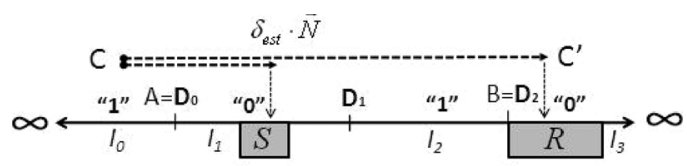

(a)

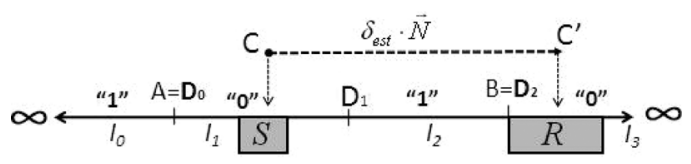

(b)

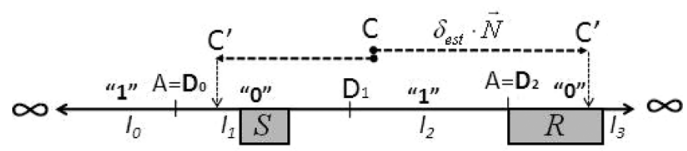

(c)

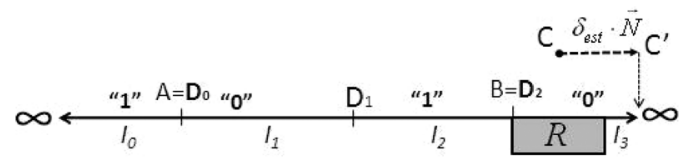

(d)

Fig. 11. Examples of core embedding process when $S_{i}=0$. Only relevant $S$ and $R$ are marked. (a) When $P(C)$ falls into $I_{0}, P(C) \notin S_{i}$. (b) When $P(C)$ falls into $I_{1}, P(C) \in S_{i}$. (c) When $P(C)$ falls into $I_{2}, P(C) \notin S_{i}$. (d) When $P(C)$ falls into $I_{3}, P(C) \in S_{i}$.

out of $I_{0}$. Watermark strength $\delta_{\text {est }}$ is determined according to the results of the psychovisual experiments. More specifically, the modified version of Cayre and Macq's method can be summarized as follows.

1) Generate a list of triangles of the mesh: The list of triangles is established as seen in Fig. 3(b). The list of triangles is stored as a secret key to the extractor. The length of the key must be the same as that of the list of admissible triangles required to convey the payload.

2) Create a new edge decomposition as seen in Fig. 9(b).

3) Perform the core embedding process as follows: 1) when $P(C) \in S_{i}$, the position of vertex $C$ is modified: it either remains in the same interval or it moves into the other interval belonging to the same $S_{i}$ set. 2) When $P(C) \notin S_{i}$, the vertex $C$ is moved into one of two intervals that do not belong to the $S_{i}$ set (see Fig. 11). Further details of this core embedding step are explained below.

4) Repeat step 3 until all the triangles on the list have been traversed.

Fig. 11 illustrates further details of the aforementioned step 3. For each interval defining $I_{0}, I_{1}, I_{2}$, and $I_{3}$, safe $(S)$ and risky $(R)$ zones are defined as depicted in Fig. 11. The size of these zones are as follows:

$$
\begin{array}{ll}
\bar{S}=\frac{1}{3}\left|I_{k}\right|, & \text { for } k=1 \text { or } 2 \\
\bar{R}=\frac{1}{2}\left|I_{k}\right|, & \text { for } k=1 \text { or } 2 .
\end{array}
$$

An $S$ zone is always located at the center of $I_{1}$ or $I_{2}$, whereas an $R$ zone is located at the (noninfinite) border of $I_{0}$ or $I_{3}$. When the embedder has to decide the new position for vertex $C$, it prefers $S$ zones and tries to avoid $R$ zones. Specifically, let us consider again the case of a " 0 " bit, i.e., $S_{i}=0$. Four possible cases can occur during watermark embedding. 
In the first case. $P(C)$ falls in $I_{0}$ [see Fig. 11(a)]. In this case, the embedder determines whether $C$ moves to $I_{1}$ or $I_{3}$ since $P(C) \notin S_{i}$. With a given watermark strength $\delta_{\text {est }}$, the embedder tries to reach $I_{3}$ while avoiding $R$. If this is not possible, the embedder moves $C^{\prime}$ into $I_{1}$, possibly by using a $\delta$ that is lower than the originally estimated one $\left(\delta_{\text {est }}\right)$. Note that when $C$ is moved into $I_{3}, C^{\prime}$ can be moved as far away from $C$ as possible within $I_{3}$. On the contrary, when $C^{\prime}$ is moved into $I_{1}$, its optimum position is to be as close to the center of $I_{1}$ as possible (i.e., the $S$ zone). However, the allowable distortion $\left(\delta_{\text {est }}\right)$ may prevent $C^{\prime}$ from being moved into the $S$ zone. In an even worse situation, it may be impossible to move $C$ outside of $I_{0}$ if $\delta_{\text {est }}$ is very small (e.g., zero roughness). This issue was resolved by adding a correction function which moves $C$ into $I_{0}$ or $I_{3}$ by recomputing $\delta_{\text {est }}$ when the value of roughness is zero. The correction function assigns a value of $\delta_{\text {est }}$ in order to allow $C$ be moved into $I_{0}$ or $I_{3}$ while avoiding the $R$ zone. Note that the magnitude of $\delta_{\text {est }}$ can be any value since perturbation occurs on a flat surface [see Fig. 5(b)].

In the second case, $P(C) \in S_{i}$ and $P(C)$ falls into $I_{1}$ [see Fig. 11(b)]. The embedder tries to move to $C$ to $I_{3}$ first by avoiding $R$. If it is successful, embedding is complete, otherwise the embedder tries to move $C$ as close as possible to the center of $I_{1}$.

In the third case, $P(C) \notin S_{i}$ and $P(C)$ falls into $I_{2}$ (see Fig. 11). In this case the embedder first tries to move $C$ toward $I_{3}$, if it fails, it tries to move $C$ into the $S$ region of $I_{1}$. If both options fail, the vertex $C$ chooses the closest one of two intervals to move in by comparing two euclidean distances, from $C$ to $I_{1}$ and to $I_{3}$, respectively.

In the last and simplest case, there is only one direction for the vertex $C$ to move within the interval $I_{3}$ to improve robustness [see Fig. 11(d)].

As explained above, it is sometimes necessary to reduce watermark strength during the embedding process in order for $C^{\prime}$ to be in an $S$ region. As a result, there is a discontinuity in the way the embedder can control the watermark strength as a function of local roughness.

\section{ROUGHNESS ADAPTIVE WATERMARKING}

By relying on the results of the psychovisual experiment described earlier, we now propose an adaptive approach to select watermark strength based on local surface roughness. Our algorithm takes advantage of the fact that the human eyes are more sensitive to distortions of smoother surface patches than to distortions of rougher surface patches. Indeed, the results of the psychovisual experiment indicated that a stronger watermark can be hidden into a bumpier surface area with a higher roughness level. Specifically, the watermark detection threshold for watermarks increases monotonically with the local surface roughness as shown in (3), (4), and (5). Our goal is to use an adaptive watermark strength determined by the local surface roughness instead of the constant watermark strength used in Benedens's method and Cayre and Macq's method. While satisfying the imperceptibility constraint, our method will result in a higher average watermark strength, that will lead to an improved robustness. Our proposed algorithm works as follows. The embedder first estimates the roughness level at each vertex.
It then chooses the maximum imperceptible watermark strength using (9) and (10)

$$
\begin{aligned}
& \delta_{\mathrm{BEN}}=\begin{array}{ll}
0.3851 * S^{0.3851}, & \text { for } S \geq 0.000082 \\
0.0001, & \text { for } S<0.000082
\end{array} \\
& \delta_{\mathrm{CAY}}=\begin{array}{ll}
0.0195 * S^{0.184}, & \text { for } S \geq 0.000082 \\
0.001, & \text { for } S<0.000082
\end{array}
\end{aligned}
$$

where $S$ denotes surface roughness. Recall that the surface roughness of the smoothest spherical surface used in the psychovisual study (see Fig. 6) was 0.000082 . For a smoother surface with roughness values lower than 0.000082 , we have heuristically set the watermark strength to a constant. In practice, however, we rarely expect to encounter a surface roughness value as low as 0.000082 for most 3 -D surfaces.

To estimate the local surface roughness around a to-be-modified vertex, the embedder estimates the roughness of all adjacent faces around the vertex using the one-ring roughness estimation method described in Corsini et al. [10]. The value of $\delta$ is then determined by (9) or (10) for the modified Benedens's method or the modified Cayre and Macq's method, respectively.

\section{Vi. Performance Evaluation of Roughness-Based ADAPTIVE WATERMARKING}

To evaluate the validity of the roughness-adaptive 3-D watermarking approach, we applied it to the modified versions of Benedens's method and Cayre and Macq's method. We focused on the demonstration of increased robustness against two types of attacks: additive noise and mesh simplification. The additive noise attack was chosen as a very general attack to evaluate the robustness of both methods, while the mesh simplification was chosen as a worst case attack against Benedens's method since 1) the mesh simplification is likely to affect the rough mesh surface more and 2) the original Benedens's method is quite powerful against mesh simplification. The additive noise attacks were generated with a Gaussian distribution and all the surface vertices were altered by the noise. The mesh simplification attacks were simulated by a quadric-based mesh decimation implemented in MeshLab software. The performance levels of our improved methods and those of the original methods were compared in terms of robustness against these attacks. Note that imperceptibility was ensured for all 3-D models used and all watermarking methods considered.

\section{A. 3-D Models}

Six 3-D models, "Angel (M1)," "Bunny1 (M2)," "Bunny2 (M3)," "Dragon (M4)," "Gorilla (M5)," and "Happy Buddha (M6)," were used for the experiments. The key characteristics of the six models are summarized in Table I. As it can be seen, the models differ in terms of resolution and surface roughness. It is expected that models with larger variations in roughness values will benefit more from our roughness-adaptive watermarking approach. The watermarked models after roughness-adaptive watermarking are shown in Fig. 12.

\section{B. Roughness Adaptive Watermarking of the 3-D Models}

Four conditions were considered in the experiments, as seen in Table II. For Condition I, the modified Benedens's method with improved robustness of " 1 " bit and blindness was used with a constant watermark strength $\delta$. The constant $\delta$ value 
TABLE I

Key Parameters of the SiX 3-D Models Used IN THE Present Study

\begin{tabular}{|c|c|c|c|c|}
\hline Model & \# of Vert. & \# of Faces & Avg. Roughness & Std. Dev. \\
\hline \hline M1 & 5002 & 10000 & $6.51 \mathrm{E}-08$ & $4.64 \mathrm{E}-08$ \\
\hline M2 & 5050 & 9999 & $1.89 \mathrm{E}-07$ & $1.75 \mathrm{E}-07$ \\
\hline M3 & 7525 & 14999 & $7.34 \mathrm{E}-08$ & $8.50 \mathrm{E}-08$ \\
\hline M4 & 3512 & 6999 & $1.33 \mathrm{E}-06$ & $8.84 \mathrm{E}-07$ \\
\hline M5 & 7521 & 14999 & $2.97 \mathrm{E}-08$ & $2.84 \mathrm{E}-08$ \\
\hline M6 & 4952 & 9932 & $7.52 \mathrm{E}-07$ & $4.48 \mathrm{E}-07$ \\
\hline
\end{tabular}

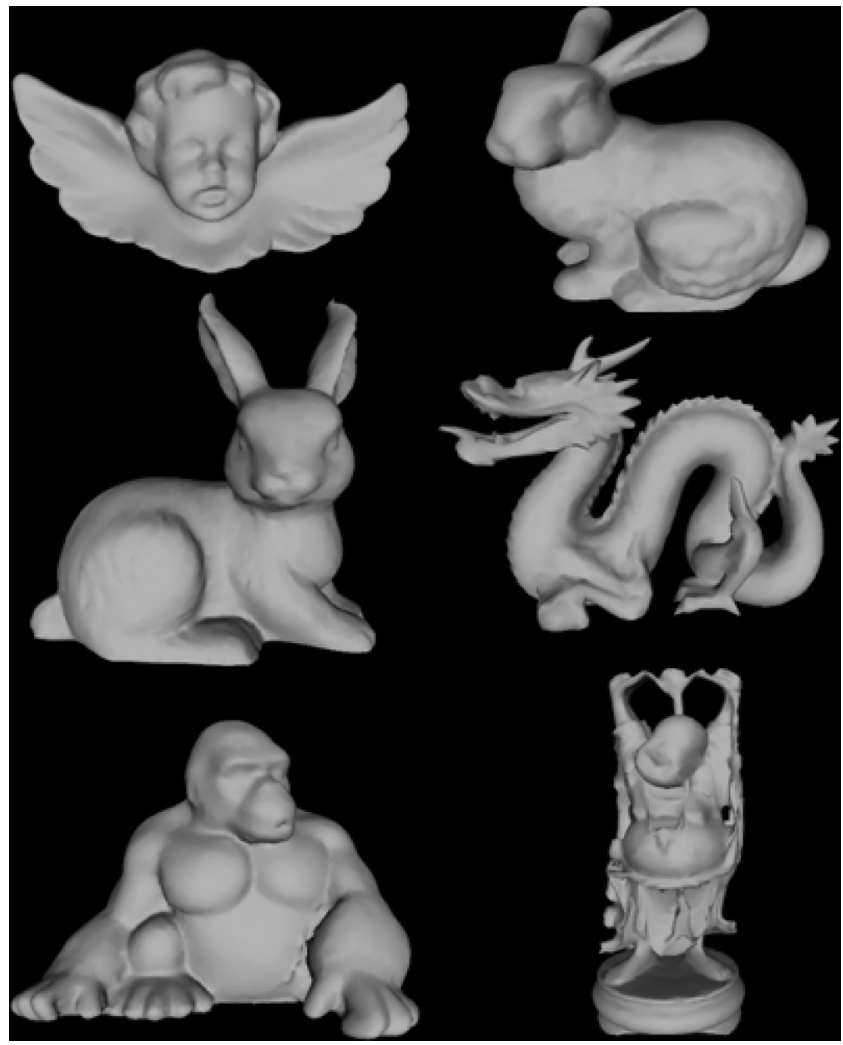

Fig. 12. Watermarked models resulting from by our roughness-adaptive approach: Angel and Bunny1 (first row), Bunny2 and Dragon (second row), and Gorilla and Happy Buddha (third row).

was chosen from a pilot test where the maximum $\delta$ value averaged from five repetitions was selected for imperceptible watermarking with each 3-D model. Condition II used the same modified version of Benedens's method with our roughness adaptive scheme. In this condition, $\delta$ was adaptively selected based on local surface roughness during the embedding process. Condition III used Cayre and Macq's method where watermark strength was determined by the type of triangles and by the order of MEP $(n=2)$. In other words, $\delta$ size varied with each of the triangles on the list of triangles to be modified and was not optimized during the embedding process. In Condition III, the $\delta$ value for each triangle was recorded and averaged to be compared with the $\delta$ values by our roughness-adaptive approach (i.e., Condition IV). Condition IV was the roughness-adaptive version of the modified Cayre and Macq's method, where $\delta$ values were adaptively selected according to local surface roughness (see also Section IV).

For Conditions I and II, the relevant parameters were set as follows: $\varphi^{R}=10^{\circ}, \alpha=10$ (heuristically chosen), 20 bins (i.e., 20 watermark bits), no $\beta$, and the entire embedding process was reiterated twice in order to get a refined $\delta$ value. The difference
TABLE II

EXPERIMENTAL CONDITIONS

\begin{tabular}{|c|c|c|}
\hline $\begin{array}{c}\text { Experimental } \\
\text { Condition }\end{array}$ & $\begin{array}{c}\text { Watermarking } \\
\text { Method }\end{array}$ & Watermark Strength $\delta$ \\
\hline \hline I & Modified Benedens & constant \\
\hline II & Modified Benedens & adaptive \\
\hline III & Cayre \& Macq & variant but not adaptive \\
\hline IV & Cayre \& Macq & adaptive \\
\hline
\end{tabular}

between Conditions I and II is related to the need of the initial watermark strength $\delta$ before starting the embedding process. For Condition I, the initial $\delta$ value was chosen by a pilot study ensuring imperceptibility. For Condition II where our roughness-adaptive approach was employed, the $\delta$ value was adaptively selected by applying (9).

To test robustness against additive noise for all conditions, Gaussian random noise $(N(0, \sigma=0.0005))$ was added to the watermarked model in order to randomly alter the locations of all vertices in the model. The bits of the embedded watermarks were extracted and compared with the original ones. Error rate was computed in terms of the percentage of mismatched bits. The experiment with each method was repeated ten times. At each iteration, a new set of Gaussian noise, watermark bits, and bins to be watermarked are selected randomly.

Additional experiments to test robustness against mesh simplification for Conditions I and II were conducted to see how much our approach can improve Benedens's method against mesh simplification. For this evaluation, a quadric-based mesh decimation implemented in MeshLab, an open source software, was used for all models with a parameter specifying the amount of mesh reduction (e.g., 80\% and 90\%). The amount of mesh reduction was calculated based on the number of faces of each model. For example, the number of faces for M1 reduced from 10000 to 2000 after $80 \%$ mesh simplification.

For Conditions III and IV, a list of 300 triangles was randomly generated for each model. The order of MEP was experimentally chosen over all six models for Condition III because the MEP's order determined the interval size for the decomposition of each triangle, which affected the amount of actual visual distortions. During the embedding process for both Conditions III and IV, the magnitudes of the modification introduced by the watermarking process for each triangle were recorded and averaged for each model. Robustness evaluation was conducted in the same way as with the modified Benedens's method. The experiments were also repeated ten times for each model.

\section{Procedures}

Watermarking methods were implemented using $\mathrm{C}++$ with CGAL and OpenGL libraries for PCs running the Windows environment. Five PCs with processing speeds from 2.4 to $3 \mathrm{GHz}$ were used. Each PC was equipped with a 17-in TFT PC monitor. The 3-D models were graphically displayed using the Gouraud shading technique [21]. On each PC, watermarks were embedded into one of the models under all four conditions.

\section{Results}

The results of the evaluation experiments are shown in Tables III, VII, VIII (for Conditions I and II) and Table IV (for Conditions III and IV). It is clear that watermarks embedded by using roughness-based adaptation (Conditions II and IV) 
TABLE III

COMPARISON OF WATERMARK DECODING ERRORS DUE TO ADDITIVE-NOISE ATTACKS FOR THE MODIFIED BENEDENS'S METHOD (CONDITIONS I AND II). THE AVERAGE AND tHE STANDARD DEVIATION OF THE ERROR RATE FOR EACH MODEL ARE SHOWN FOR EACH CONDITION. THE IMPROVEMENT IS SHOWN AS THE REDUCTION IN ERROR RATE IN PERCENTAGE

\begin{tabular}{|c|c|c|c|}
\hline Model & Condition I (\%) & Condition II (\%) & Improvement (\%) \\
\hline \hline M1 & $10 \pm 3.16$ & $4.5 \pm 3.69$ & 55.0 \\
\hline M2 & $9.5 \pm 3.50$ & $4 \pm 2.11$ & 57.9 \\
\hline M3 & $9 \pm 3.74$ & $4 \pm 3.16$ & 55.5 \\
\hline M4 & $10 \pm 3.47$ & $2.5 \pm 2.64$ & 75.0 \\
\hline M5 & $9.5 \pm 2.69$ & $4.5 \pm 2.84$ & 52.6 \\
\hline M6 & $9.5 \pm 4.15$ & $3.5 \pm 2.42$ & 63.2 \\
\hline
\end{tabular}

are more robust against additive-noise and mesh simplification attacks for all the six models.

For additive noise attacks, the robustness improvements achieved by our method (Condition II) from the modified version of Benedens's method ranged from $52.6 \%$ with the Gorilla (M5) to $75 \%$ with the Dragon model (M4). The improvements in robustness over Cayre and Macq's method ranged from $45.6 \%$ with the Angel model (M1) to $61.6 \%$ with the Dragon model (M4) (see Table IV). As expected, the largest improvement occurred with the Dragon model because the standard deviation of surface roughness for the Dragon model (M4) was the largest among the six models tested (see Table I).

It was also found (see Tables V and VI) that the average value of $\delta$ was larger with our roughness-adaptive methods (Conditions II and IV) than with the modified Benedens's method (Condition I) and Cayre's and Macq's method (Condition III). The increase in $\delta$ was greater with the Dragon model (M4) and the Happy Buddha model (M6) than that with the other models. Therefore, as we expected, the models with the larger variations in surface roughness (the Dragon model) benefited more from roughness-adaptive watermarking.

For mesh simplification attacks, the robustness improvements achieved by our method ranged from $26 \%$ with the Dragon modal (M4) to $40 \%$ with the Bunny2 (M3) and Gorilla (M5) models as $80 \%$ of faces of the original mesh was reduced, and ranged from $9.1 \%$ with the Dragon model (M4) to $25.93 \%$ with the Gorilla model (M5) as $90 \%$ of faces of the original mesh was reduced (see Tables VII and VIII). As for additive noise roughness adaptive watermarking still results in increased robustness, however the improvement pattern is considerably different than for the additive noise attack over the six models.

\section{E. Discussions}

In this paper, we described our roughness-adaptive 3-D watermarking approach based on the masking effect of surface roughness on embedded watermarks. We applied our approach to two existing watermarking schemes by Benedens and by Cayre and Macq. Our results demonstrated improved watermark robustness in all cases tested.

Our study measured the human watermark detection thresholds as a function of surface roughness, and uses the values as upper bounds for assigning watermark strengths locally based on surface roughness. This approach ensured that the watermarks embedded by our approach are guaranteed to be imperceptible under any circumstances. For this reason, there is no need to evaluate the invisibility of watermarks embedded with our approach. To highlight this point, we show two watermarked
TABLE IV

COMPARISON OF WATERMARK DECODING ERRORS DUE TO ADDITIVE-NOISE ATTACKS FOR CAYRE AND MACQ'S METHOD (CONDITIONS III AND IV). THE AVERAGE AND THE STANDARD DEVIATION OF THE ERROR RATE FOR EACH MODEL ARE SHOWN FOR EACH CONDITION. THE IMPROVEMENT IS SHOWN AS THE REDUCTION IN ERROR RATE IN PERCENTAGE

\begin{tabular}{|c|c|c|c|}
\hline Model & Condition III (\%) & Condition IV (\%) & Improvement (\%) \\
\hline \hline M1 & $42.9 \pm 1.49$ & $23.3 \pm 3.49$ & 45.6 \\
\hline M2 & $48.3 \pm 4.58$ & $22.8 \pm 3.41$ & 52.6 \\
\hline M3 & $47.4 \pm 2.85$ & $24.5 \pm 4.52$ & 48.8 \\
\hline M4 & $46.8 \pm 4.67$ & $18.0 \pm 1.93$ & 61.6 \\
\hline M5 & $45.5 \pm 2.48$ & $24.1 \pm 2.58$ & 46.9 \\
\hline M6 & $44.0 \pm 3.15$ & $19.6 \pm 3.67$ & 55.5 \\
\hline
\end{tabular}

TABLE V

COMPARISON OF WATERMARK STRENGTHS FOR THE MODIFIED BENEDENS'S METHOD (CONDITIONS I AND II). ALl Values ARE SCALED DOWN By 1000

\begin{tabular}{|c|c|c|c|}
\hline Model & Condition I & Condition II & Improvement (\%) \\
\hline \hline M1 & $1.14 \pm 0.13$ & $2.06 \pm 0.48$ & 80.4 \\
\hline M2 & $1.05 \pm 0.066$ & $2.65 \pm 0.57$ & 151.7 \\
\hline M3 & $1.06 \pm 0.022$ & $1.98 \pm 0.65$ & 85.45 \\
\hline M4 & $1.29 \pm 0.098$ & $8.21 \pm 0.79$ & 534.8 \\
\hline M5 & $1.09 \pm 0.086$ & $1.94 \pm 0.88$ & 77.5 \\
\hline M6 & $1.03 \pm 0.014$ & $4.79 \pm 0.64$ & 364.9 \\
\hline
\end{tabular}

TABLE VI

COMPARISON OF WATERMARK STRENGTHS FOR CAYRE AND MACQ'S METHOD (Conditions III AND IV). All Values are SCALEd Down By 1000

\begin{tabular}{|c|c|c|c|}
\hline Model & Condition III & Condition IV & Improvement (\%) \\
\hline \hline M1 & $1.15 \pm 0.21$ & $4.64 \pm 0.35$ & 300.2 \\
\hline M2 & $1.17 \pm 0.71$ & $4.88 \pm 0.48$ & 318.9 \\
\hline M3 & $1.17 \pm 0.10$ & $4.78 \pm 0.49$ & 309.2 \\
\hline M4 & $1.16 \pm 0.59$ & $5.90 \pm 0.97$ & 409.6 \\
\hline M5 & $1.12 \pm 0.27$ & $4.49 \pm 0.37$ & 302.4 \\
\hline M6 & $1.20 \pm 0.26$ & $5.73 \pm 0.39$ & 357.1 \\
\hline
\end{tabular}

TABLE VII

COMPARISON OF Watermark DECODING ERRORS DUE TO MESH SIMPLIFICATION ATTACKS (80\% REDUCTION) FOR BENEDENS's METHOD (CONDITIONS I AND II)

\begin{tabular}{|c|c|c|c|}
\hline Model & Condition I (\%) & Condition II (\%) & Improvement (\%) \\
\hline \hline M1 & $9 \pm 3.74$ & $5.5 \pm 4.38$ & 38.9 \\
\hline M2 & $10.5 \pm 4.15$ & $6.5 \pm 2.42$ & 38.1 \\
\hline M3 & $5 \pm 3.16$ & $3 \pm 2.60$ & 40 \\
\hline M4 & $32 \pm 6.40$ & $23.5 \pm 4.74$ & 26.6 \\
\hline M5 & $2.5 \pm 2.5$ & $1.5 \pm 2.41$ & 40 \\
\hline M6 & $30 \pm 7.75$ & $22 \pm 5.87$ & 26.7 \\
\hline
\end{tabular}

models (Bunny1) by the modified Benedens's method as seen in Fig. 13. On the left [Fig. 13(a)], watermark strength was adaptively chosen according to the perception curve and it is apparent that the watermarks were invisible. On the right [Fig. 13(b)], the distortion introduced by watermarks became visible when a constant watermark strength, equal to the average watermark strength used for our roughness-adaptive watermarking, was used. This example shows the superiority, in terms of imperceptibility, of our roughness-adaptive approach to the original Benedens's method where a constant watermark strength was used.

From our results (Tables III, V, IV, VI, VII, and VIII), it can be clearly stated that roughness adaptive watermarking employing human sensitivity to local surface roughness significantly improves overall watermark strength, leading to superior 
TABLE VIII

COMPARISON OF WATERMARK DECODING ERRORS DUE TO MESH SimPLiFICATION ATTACKS (90\% REDUCTION) FOR BENEDENS's METHOD (CONDITIONS I AND II)

\begin{tabular}{|c|c|c|c|}
\hline Model & Condition I (\%) & Condition II (\%) & Improvement (\%) \\
\hline \hline M1 & $25 \pm 5.0$ & $21 \pm 5.68$ & 16 \\
\hline M2 & $28.5 \pm 5.94$ & $23 \pm 4.83$ & 19.3 \\
\hline M3 & $18 \pm 5.1$ & $15 \pm 3.3$ & 16.7 \\
\hline M4 & $44 \pm 8.9$ & $40 \pm 5.8$ & 9.1 \\
\hline M5 & $13.5 \pm 5.0$ & $10 \pm 4.1$ & 25.93 \\
\hline M6 & $45.5 \pm 9.6$ & $40.5 \pm 7.98$ & 10.99 \\
\hline
\end{tabular}

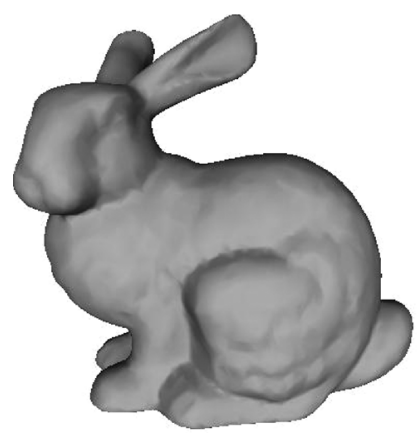

(a)

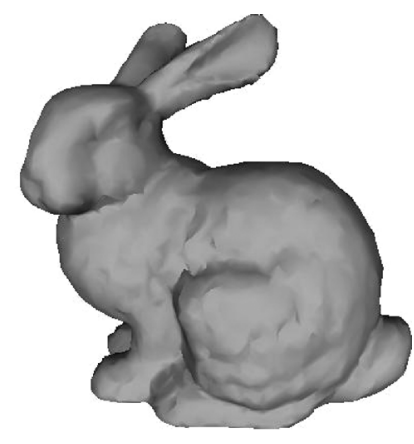

(b)
Fig. 13. Comparison of invisibility of embedded watermarks by the modified Benedens's method using 1) adaptive and 2) constant watermark strengths. (a) Adaptive $\delta=0.00265$ (average). (b) Constant $\delta=0.00265$.

robustness against attacks. The results with both Benedens's method and Cayre and Macq's method strongly support the statement although the impact of the roughness-adaptive watermarking approach varies with the characteristics of input models, with models having larger surface roughness variations benefiting more from this approach.

As seen in Tables V and VI, overall $\delta$ was increased more with Cayre and Macq's method than with Benedens's method. One reason for this difference is that people are more sensitive to perturbations with Benedens's method (hence a lower watermark detection threshold) than with Cayre and Macq's method (see Fig. 8). As a result, the watermark embedder was able to increase watermark strength more with Cayre and Macq's method when utilizing the roughness-adaptive approach. Another reason is that the maximized $\delta$ values in the case of zero surface roughness with Cayre and Macq's method contributed to a further increase of the overall watermark strength as compared to Benedens's method. An exception to this general trend is found in the fourth row (M4: Dragon model) of Tables V and VI, where the increase in watermark strength with Benedens's method is larger than that with Cayre and Macq's method. The reason is that the constraint of the modified Cayre and Macq's method (discontinuity of watermark strength described at the end of Section IV) resulted in a reduction of the maximized watermark strength.

The results against mesh simplification, seen in Tables VII and VIII, show a different pattern from additive noise attacks in terms of improvement in robustness over the six models because of the characteristics of mesh simplification. Mesh simplification is one of the worst conceivable attacks since it affects the original topology that was used for optimizing watermark strengths during embedding. For this reason, the improved robustness against mesh simplification that we have measured in our experiments is particularly significant and demonstrates the validity of the roughness-adaptive approach.

\section{CONCLUSION}

Developing robust 3-D digital watermarking techniques is an ongoing challenging research topic in the field of information hiding. In this paper, we have presented a general way to improve watermark robustness by exploiting masking effects of human visual perception. Our method is based on a measure of human sensitivity to surface variations as a function of surface roughness of input meshes, ensuring imperceptibility of embedded watermarks. We emphasize that the beauty of our approach is that once we have obtained human detection thresholds (Fig. 8) from our psychovisual study (Section III), there is no longer the need to worry about invisibility.

The evaluation experiments in which we applied our roughness-adaptive scheme to two existing 3-D watermarking methods by Benedens and by Cayre and Macq confirmed that the overall watermark robustness is improved significantly as a result of increased watermark strengths through roughness-adaptive watermark embedding. As expected, the experiments demonstrated that the roughness-adaptive watermarking technique brings about more benefits to data models with larger standard deviations of surface roughness levels. We showed that, on average, stronger watermarks can be embedded with roughness-adaptive watermark strengths than could be achieved with a constant watermark strength as used by most watermarking methods.

By combining our results with the results of Uccheddu et al. [11] who utilized surface roughness in the spectral domain, we can make a statement that utilizing masking effect due to surface roughness of polygonal meshes is an effective way to improve watermark robustness while maintaining watermark imperceptibility. Therefore, our approach suggests promising new directions for improving the performance of any type of 3-D digital watermarking schemes. In the future, we will continue to evaluate our roughness-adaptive scheme with additional 3-D models. We also plan to investigate masking effects characterized by other geometric properties such as 3-D curvatures. Our ultimate goal is to explore the masking property of human visual system as a general strategy for improving 3-D digital watermarking techniques.

\section{ACKNOWLEDGMENT}

The authors would like to thank J. Park for providing some of the 3-D models used for their experiments.

\section{REFERENCES}

[1] O. Benedens, "Geometry-based watermarking of 3D models," IEEE Comput. Graph. Appl., vol. 19, no. 1, pp. 46-55, Jan./Feb. 1999.

[2] F. Cayre and B. Macq, "Data hiding on 3-D triangle meshes," IEEE Trans. Signal Process., vol. 51, no. 4, pp. 939-949, Apr. 2003.

[3] R. Ohbuchi, H. Masuda, and M. Aono, "Watermaking three-dimensional polygonal models," in Proc. Fifth ACM Int. Conf. Multimedia, Seattle, WA, 1997, pp. 261-272. 
[4] F. Bartolini, M. Barni, V. Cappellini, and A. Piva, "Mask building for perceptually hiding frequency embedded watermarks," in Proc. 1998 Int. Conf. Image Processing, 1998 (ICIP 98), Oct. 1998, vol. 1, pp. $450-454$.

[5] M. Barni, F. Bartolini, and A. Piva, "Improved wavelet-based watermarking through pixel-wise masking," IEEE Trans. Image Process., vol. 10, no. 5, pp. 783-791, May 2001.

[6] I. J. Cox and M. L. Miller, "Review of watermarking and the importance of perceptual modeling," Proc. SPIE, vol. 3016, no. 1, pp. 92-99.

[7] R. B. Wolfgang, C. I. Podilchuk, and E. J. Delp, "Perceptual watermarks for digital images and video," in Proc. Society of Photo-Optical Instrumentation Engineers (SPIE) Conf. Series, Apr. 1999, vol. 3657, pp. $40-51$.

[8] C. Podilchuk and W. Zeng, "Perceptual watermarking of still images," in IEEE First Workshop on Multimedia Signal Processing, 1997, Jun. 1997, pp. 363-368.

[9] C. Podilchu and W. Zeng, "Image-adaptive watermarking using visual models," IEEE J. Sel. Areas Commun., vol. 16, no. 4, pp. 525-539, May 1998.

[10] M. Corsini, E. D. Gelasca, T. Ebrahimi, and M. Barni, "Watermarked 3-D mesh quality assessment," IEEE Trans. Multimedia, vol. 9, no. 2, pp. $247-256$, Feb. 2007

[11] F. Uccheddu, M. Corsini, M. Barni, and V. Cappellini, "A roughnessbased algorithm for perceptual watermarking of 3D meshes," in Proc. 10th Int. Conf. Virtual System and Multimedia, 2004, pp. 934-943.

[12] O. Benedens, "Watermarking of 3D polygon based models with robustness against mesh simplification," in Proc. SPIE: Security and Watermarking of Multimedia Contents, 1999, pp. 329-340.

[13] J. A. Ferwerda, P. Shirley, S. N. Pattanaik, and D. P. Greenberg, "A model of visual masking for computer graphics," in Proc. 24th Annи. Conf. Computer Graphics and Interactive Techniques (SIGGRAPH '97), New York, 1997, pp. 143-152.

[14] S. Kanai, H. Date, and T. Kishinami, "Digital watermarking for 3D polygons using multiresolution wavelet decomposition," in Proc. IFIP WG, 1998, pp. 296-307.

[15] A. Bors, "Watermarking mesh-based representations of 3-D objects using local moments," IEEE Trans. Image Process., vol. 15, no. 3, pp. 687-701, Mar. 2006.

[16] F. Uccheddu, M. Corsini, and M. Barni, "Wavelet-based blind watermarking of 3D models," in Proc. 2004 Workshop on Multimedia and Security (MM\&Sec '04), 2004, pp. 143-154.

[17] M. Lounsbery, T. D. DeRoseJ, and Warren, "Multiresolution analysis for surfaces of arbitrary topological type," ACM Trans. Graph., vol. 16, no. 1, pp. 34-73, 1997.

[18] K. Kim, M. Barni, and H. Z. Tan, "Roughness-adaptive 3D watermarking of polygonal meshes," in Proc. Information Hiding: 11th Int. Workshop, Darmstadt, Germany, Jun. 8-10, 2009, pp. 191-205.

[19] J. Nelder and R. Mead, "A simplex method for function minimization," Comput. J., vol. 7, no. 4, pp. 308-313, 1965.

[20] H. Levitt, "Transformed up-down methods in psychoacoustics," $J$. Acoustical Soc. Amer., vol. 49, no. 2B, pp. 467-477, 1971.

[21] H. Gouraud, "Continuous shading of curved surfaces," IEEE Trans. Comput., vol. C-20, no. 6, pp. 623-629, Jun. 1971.

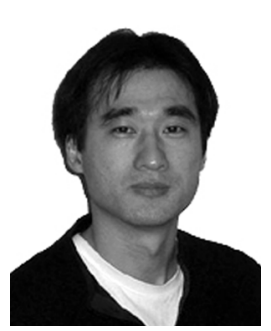

Kwangtaek Kim (S'10-M'10) received the Bachelor's and Master's degrees in electronic engineering from Korea University in 1998 and 2001, respectively. Since August 2005, he has been working toward the Ph.D. degree in the School of Electrical and Computer Engineering, Purdue University, West Lafayette, IN.

He has worked as a research engineer in the fields of biomedical imaging and digital image compression for several companies in Korea. His research interests include 3-D digital watermarking, visuohaptic rendering, psychophysics, digital image processing, and computer vision.

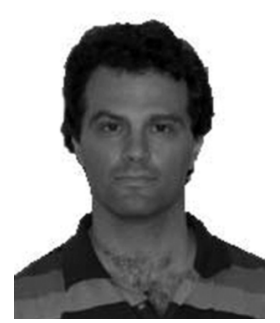

Mauro Barni (S'88-M'96-SM'06) received the Bachelor's degree in electronic engineering from the University of Florence in 1991. He received the Ph.D. degree in informatics and telecommunications in October 1995.

He has carried out his research activity for over 18 years first at the Department of Electronics and Telecommunication, the University of Florence, Florence, Italy, then at the Department of Information Engineering, University of Siena, Siena, Italy, where he works as Associate Professor. During the last decade, he has been studying the application of image processing techniques to copyright protection and authentication of multimedia (digital watermarking) $\mathrm{He}$ is author/coauthor of about 200 papers published in international journals and conference proceedings, and holds three patents in the field of digital watermarking. He is coauthor of the book Watermarking Systems Engineering: Enabling Digital Assets Security and other Applications (Dekker Inc., 2004).

Dr. Barni participated in several National and European research projects on diverse topics, including computer vision, multimedia signal processing, remote sensing, digital watermarking, and IPR protection. In particular, he is the coordinator of the project SPEED (Signal Processing in the EncryptEd Domain), funded by the EC under the FP6 (FET - program). He is the editor-in-chief of the EURASIP Journal on Information Security. He serves as associate editor of the IEEE TRANSACTIONS ON CIRCUITS AND SYSTEMS FOR VIDEO TECHNOLOGY and the IET Proceedings on Information Security. He was the general chairman of the 2004 edition of IEEE Workshop on Multimedia Signal Processing (MMSP'04) and the 2005 edition of the International Workshop on Digital Watermarking (IWDW'05). He is the chairman of the IEEE Information Forensics and Security Technical Committee (IFS-TC) of the IEEE Signal Processing Society. He is a senior member of EURASIP.

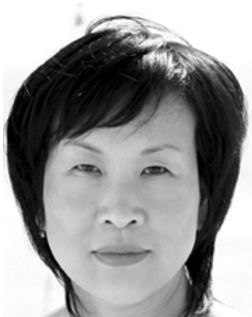

Hong Z. Tan (S'96-M'96-SM'06) received the Bachelor's degree in biomedical engineering (in 1986) from Shanghai Jiao Tong University and the Master's and Doctorate degrees (in 1988 and 1996 respectively), both in electrical engineering and computer science, from the Massachusetts Institute of Technology (MIT).

She was a Research Scientist at the MIT Media Laboratory from 1996 to 1998, before joining the faculty at Purdue University, West Lafayette, IN. She is currently an associate professor of electrical and computer engineering, with courtesy appointments in the School of Mechanical Engineering and the Department of Psychological Sciences. She has published more than 110 peer-reviewed articles in journals and conference proceedings and two book chapters. Her research focuses on haptic human-machine interfaces in the areas of haptic perception, rendering, and multimodal performance.

Dr. Tan is an associate editor of Presence, ACM Transactions on Applied Perception, and IEEE TRANSACTIONS ON HAPTICS. She was a co-organizer (with Blake Hannaford) of the International Symposium on Haptic Interfaces for Virtual Environment and Teleoperator Systems from 2003 to 2005. She served as the founding chair of the IEEE Technical Committee on Haptics, a home for the international interdisciplinary haptics research community, from 2006 to 2008 She was a recipient of the National Science Foundation CAREER award from 2000 to 2004, and a coauthor of "Haptic feedback enhances force skill learning" which won the best paper award at the 2007 World Haptics Conference. She is a member of the Psychonomic Society. 